\title{
Les représentations de la féminité à travers les adaptations de Nana d'Émile Zola pour le petit et le grand écran
}

\author{
Charlotte Andrieux \\ Association des Amis de Roger Martin du Gard \\ chandrieux@aol.com
}

Rebut: 20 de desembre de 2014

Acceptat: 30 me març de 2015

RESUM

Representacions de la feminitat a través de les adaptacions de Nana d'Emile Zola a la petita i gran pantalla

Aquest article es proposa estudiar les representacions de la feminitat a través del personatge de Nana, comparant una selecció de sis adaptacions cinematogràfiques i televisives de la cèlebre novel-la epònima d'Emile Zola, realitzades entre 1926 i 2001 per Jean Renoir, Dorothy Arzner, ChristianJaque, Maurice Cazeneuve, Dan Wolman i Edouard Molinaro.

Deixant enrere una perspectiva clàssica que es basaria només en criteris de fidelitat a l'obra original i en el valor artístic de la recreació, aquesta anàlisi, fonamentada en tesis de diversos teòrics de l'adaptació cinematogràfica, intenta discernir el que aquestes noves relectures de la novel-la li aporten quan la transformen o renoven a través de mecanismes de supressió, condensació, aportacions, transposicions o transformacions més o menys exigits pel procés de transmediació, o pels objectius propis dels cineastes.

El nostre propòsit consisteix a determinar en quina mida aquestes reinterpretacions donen forma a un mite de la feminitat en constant evolució, o més precisament a l'evolució d'una dona que, segons les èpoques i els països, ha perseguit una forma d'independència basada sobretot en els seus atributs femenins, no tant per a dominar el gènere masculí sinó per a aconseguir igualar-lo. 


\section{Mots Clau}

Emile Zola, Nana, Jean Renoir, Dorothy Arzner, Christian-Jaque, Maurice Cazeneuve, Dan Wolman, Edouard Molinaro, Cinema i Literatura, Novel.la popular, Adaptacions cinematogràniques, Adaptacions televisives, Teories de l'adaptació, Condensació, Supressió, Transposició, Transmediació, Fidelitat, valors artístics i estètics, Escriptura cinematogràfica, Femení, Feminitat, Feminisme, Sexe, Cortesana, Prostituta, Desig, Voluptat, Lesbianisme, Fantasmes masculins, Estereotips femenins.

\section{RÉSUMÉ}

\section{Les représentations de la féminité à travers les adaptations de Nana d'Emile Zola pour le petit et le grand écran}

Cet article propose d'étudier les représentations de la féminité à travers le personnage de Nana, en comparant une sélection de six adaptations cinématographiques et télévisuelles du célèbre roman éponyme d'Emile Zola, réalisées de 1926 à 2001 par Jean Renoir, Dorothy Arzner, Christian Jaque, Maurice Cazeneuve, Dan Wolman et Edouard Molinaro.

Au-delà d'une perspective classique qui serait uniquement liée aux critères de la fidélité à l'œuvre originelle et à la valeur artistique de la recréation, cette réflexion, qui s'appuie sur les recherches de divers théoriciens de l'adaptation cinématographique, tente de discerner ce que ces nouvelles relectures du roman apportent à ce dernier, en le renouvelant ou le transformant, par des opérations de suppression, de condensation, d'ajouts, de transpositions ou de transformations, plus ou moins guidées par les principes mêmes du processus de transmédiation, ou bien par les intentions personnelles des réalisateurs.

L'objectif étant de déterminer en quoi ces diverses réinterprétations donnent forme à un mythe de la féminité en constante évolution, ou plus exactement au parcours d'une femme qui, selon les époques et les pays, a tenté d'acquérir une forme d'indépendance, principalement grâce à ses atouts féminins, non pas tant pour dominer le genre masculin que pour parvenir à l'égaler.

\section{Mots Clés}

Emile Zola, Nana, Jean Renoir, Dorothy Arzner, Christian-Jaque, Maurice Cazeneuve, Dan Wolman, Edouard Molinaro, Cinéma et Littérature, Roman populaire, Adaptations cinématographiques, Adaptations télévisuelles, Théories de l'adaptation, Condensation, Suppression, Transposition, Transmédiation, Fidélité, valeurs artistique et esthétique, Ecriture cinématographique, Féminin, Féminité, Féminisme, Sexe, Courtisane, Prostituée, Désir, Volupté, Lesbianisme, Fantasmes masculins, Stéréotypes féminins. 


\section{RESUMEN}

Representaciones de la feminidad a través de las adaptaciones de Nana de Emile Zola a la pequeña y la gran pantalla

Este artículo se propone estudiar las representaciones de la feminidad a través del personaje de Nana, comparando una selección de seis adaptaciones cinematográficas y televisivas de la célebre novela epónima de Émile Zola, realizadas entre 1926 y 2001 por Jean Renoir, Dorothy Arzner, ChristianJaque, Maurice Cazeneuve, Dan Wolman y Edouard Molinaro.

Más allá de una perspectiva clásica que atendería únicamente a criterios de fidelidad a la obra original y al valor artístico de la recreación, este análisis, basado en tesis de diversos teóricos de la adaptación cinematográfica, intenta discernir lo que estas nuevas relecturas de la novela le aportan, al transformarlo o renovarlo, a través de mecanismos de supresión, condensación, aportaciones, transposiciones o transformaciones más o menos exigidas por el proceso de transmediación, o por las metas propias de los cineastas.

Nuestro objetivo consiste en determinar en qué medida estas reinterpretaciones dan forma a un mito de la feminidad en constante evolución, o más precisamente a la evolución de una mujer que, según las épocas y los países, ha perseguido una forma de independencia basada sobre todo en sus atributos femeninos, no tanto para dominar al género masculino sino para lograr igualarlo.

\section{Palabras Clave}

Emile Zola, Nana, Jean Renoir, Dorothy Arzner, Christian-Jaque, Maurice Cazeneuve, Dan Wolman, Edouard Molinaro, Cine y Literatura, Novela popular, Adaptaciones cinematográficas, Adaptaciones televisivas, Teorías de la adaptación, Condensación, Supresión, Transposición, Transmediación, Fidelidad, valores artísticos y estéticos, Escritura cinematográfica, Femenino, Feminidad, Feminismo, Sexo, Cortesana, Prostituta, Deseo, Placer, Lesbianismo, Fantasmas masculinos, Estereotipos femeninos.

\section{ABSTRACT}

The representations of femininity in the adaptations of Emile Zola's Nana for the small and big screen

This article proposes to examine and compare the representations of the femininity through the character of Nana in a selection of six film and television adaptations from the Emile Zola's famous eponym novel, realized from 1926 till 2001 by Jean Renoir, Dorothy Arzner, ChristianJaque, Maurice Cazeneuve, Dan Wolman, et Edouard Molinaro.

Beyond a classic perspective, which would be only guided by the criteria of the fidelity to the original creation and by the artistic value of the re-creation, 
this reflection, which is based on the researches of many theorists of film adaptations, aims to discern what these new re-reading of the novel bring to the original creation, by renewing or transforming it, by operations of deletion, condensation, addition, transposition or transformation, more or less guided by the principles of the Transmediation process, either than by the personal intentions of the various directors.

The objective was to determine how these new interpretations create a constantly evolving myth of the femininity, or more exactly the way of a woman who, according to times and countries, tried to acquire a kind of independence, mainly thanks to its feminine assets, not in order to dominate the masculine gender but to succeed in equaling it.

\section{KEYWORDS}

Emile Zola, Nana, Jean Renoir, Dorothy Arzner, Christian-Jaque, Maurice Cazeneuve, Dan Wolman, Edouard Molinaro, Cinema and Literature, Popular Novel, Film Adaptations, Television Adaptations, Theories of the Adaptation, Condensation, Deletion, Transposition, Transmediation, Fidelity, Artistic and esthetic values, Film writing, Feminine, Femininity, Feminism, Sex, Courtesan, Prostitute, Desire, Sensual Delight, Lesbianism, Male Fantasies, Feminine Stereotypes.

Depuis les débuts du cinématographe jusqu'à nos jours et dans les pays les plus divers, les romans de Zola ont donné lieu à d'incalculables adaptations, d'abord cinématographiques puis télévisuelles, qui ont elles mêmes fait l'objet d'une grande variété d'études, aussi bien dans le domaine de la recherche littéraire que de l'étude filmique. Un phénomène d'une telle ampleur a plusieurs explications.

D'un point de vue financier, les producteurs misent souvent sur l'adaptation de romans populaires, anticipant un succès d'audience équivalent, voire supérieur à celui des ventes de l'ouvrage, puisque que l'on parie à juste titre sur le fait les spectateurs seront toujours plus nombreux que les lecteurs. Or les romans de Zola sont «populaires » à double titre : ce sont tout d'abord des romans dont les personnages sont principalement issus du peuple, et qui ont pu être lus par le peuple (via leur diffusion par la presse sous forme de «feuilletons $\left.»^{1}\right)$.

\footnotetext{
${ }^{1}$ Le roman a d'abord été publié dans Le Voltaire du 16 octobre 1879 au 5 février 1880, puis en volume chez Charpentier, le 14 février 1880.
} 
Une explication d'une autre nature, mais tout aussi déterminante, qui revient souvent dans les études sur les adaptations des romans de Zola, est liée aux spécificités de l'écriture de ce romancier, dont on connait l'intérêt pour la photographie. Beaucoup de spécialistes de Zola décèlent en effet chez lui une écriture que l'on pourrait qualifier de visuelle, cinématographique ou encore « scénarique », selon la terminologie choisie ${ }^{2}$. De ce fait, ses œuvres seraient particulièrement propices à la «transmédiation». Bien qu'il faille selon nous sans doute nuancer cette affirmation, car les difficultés de transpositions rencontrées par les scénaristes adaptateurs nous montrent clairement les limites d'une écriture dite " cinématographique ».

Le roman Nana pourrait servir de cas d'école, car il représentatif de cette attractivité de l'œuvre de Zola, puisqu'il aurait donné lieu à pas moins d'une vingtaine d'adaptations d'époques et de pays très divers ${ }^{3}$. Il n'est malheureusement plus possible de visionner la totalité de ces réalisations, et dans le cadre de cette première approche, nous avons été en mesure d'avoir accès au visionnage de six films (4 films et 2 téléfilms) qui constitueront le corpus de notre réflexion : la Nana de Renoir de 1926, l'adaptation américaine de Dorothy Arzner et George Fitzmaurice ${ }^{4}$ en 1934, Nana, Lady of the boulevards, celle de Christian Jaque en 1955, le téléfilm de Maurice Cazeneuve diffusé en 1981, l'adaptation américanoitalienne de Dan Wolman en 1983, et enfin le second téléfilm d'Edouard Molinaro, dont le titre est devenu « Nadia Coupeau, dite Nana » en 2001. La distribution complète ces six adaptations est donnée à la fin de cette étude.

Nous avons choisi ce roman, car il se prêtait particulièrement bien à la réflexion qui nous préoccupe sur les « représentations de la féminités dans les

\footnotetext{
${ }^{2}$ On lira à ce sujet l'étude de Michel Larouche et Serge Cardinal, « une écriture «scénarique « », dans Zola et le cinéma, sous la direction de Paul Warren, Les Presses de l'Université de Laval ; les Presses de la Sorbonne Nouvelle, 1995, p. 316.

${ }^{3}$ La liste exhaustive des adaptations du roman que nous donnons à la fin de cette étude est sans doute incomplète et peutêtre en partie erronée, car le Guide Emile Zola (2002) dénombre 13 adaptations de 1910 à 2001, tandis qu'Anne Marie Baron en dénombre 17, en comptant la Nana de Claude Miller en 1977, interprétée par Emmanuelle Seigner, qui n'est en réalité qu'un simple projet n'ayant jamais abouti. Nous n'avons pas pu vérifier l'existence de tous les films recensés, car nos recherches auprès de l'INA (Institut national de l'audiovisuel) et du CNC (Centre national du cinéma et de l'image animée) sur le site de la Bibliothèque François Mitterrand, nous ont révélé que l'accès au visionnage de certains films s'avère extrêmement ardu, voire impossible dans certains cas de « films perdus ».

${ }^{4}$ Dans toutes les mentions de ce film, celuici est présenté comme une coréalisation, mais le nom de George Fitzmaurice n'apparaît pourtant pas au générique... Au cours de cette étude, nous ne citerons donc que Dorothy Arzner comme réalisatrice de cette adaptation.
} 
adaptations cinématographiques et télévisuelles des romans populaires ». Le personnage de Nana peut en effet être considéré comme le «stéréotype » de la féminité, stéréotype certes réducteur, puisqu'elle ne représente pas l'ensemble du genre féminin, mais un type de femme dont les principales caractéristiques sont liées à ses atouts prétendument spécifiques à son sexe. Si Zola avait voulu représenter une « cococotte », une "prostituée », une "courtisane artiste », nous verrons que dans les adaptations, le thème de la prostitution est souvent largement atténué. En revanche, Nana demeure une artiste de peu de talent, qu'elle soit chanteuse, figurante, danseuse, meneuse de revue, actrice de film érotique ou de film publicitaire...

Nous nous demanderons donc en quoi les adaptations reprennent, retranscrivent ou transforment cette image de la femme légère devenue mythique, depuis l'ascension et la chute d'une courtisane, jusqu'au combat d'une féministe plus ou moins assumée, selon les lectures personnelles des réalisateurs successifs. Flaubert avait parfaitement saisi ce potentiel que possédait l'héroïne de Zola, comme il l'écrivait à ce dernier, le 15 février 1880 : « Nana tourne au mythe sans cesser d'être réelle. Cette création est babylonienne »!

Plusieurs chercheurs s'étaient déjà penchés sur l'image de la femme et du féminin dans l'œuvre de Zola, et quelques études ponctuelles traitent des adaptations de Nana pour le petit et le grand écran, mais cellesci prennent rarement en compte simultanément plusieurs adaptations (télévisuelles ou cinématographiques), quelle que soit leur qualité, pour adopter un point de vue comparatif. De plus, c'est bien entendu le chef d'œuvre de Renoir qui est le plus souvent, voire quasi exclusivement étudié. Il faut toutefois souligner qu'AnneMarie Baron aborde assez brièvement, mais de manière très pertinente, cette question dans un chapitre intitulé «Nana, cet objet obscur du plaisir » de son étude générale consacrée aux Romans du XIXe siècle à l'écran ${ }^{5}$.

Les six adaptations que nous avons examinées se sont avérées constituer un corpus extrêmement riche, complexe et varié, bien que partiel, qui offre finalement un éventail exhaustif des différents types d'adaptations, sur lesquelles les théoriciens du cinéma se sont longuement interrogés, dans l'intention de les classifier et de les évaluer, selon divers critères plus ou moins subjectifs. Adaptations, que l'on considère tantôt comme de simples illustrations, des transpositions, ou bien de nouvelles créations constituant des œuvres personnelles, à part entière.

\footnotetext{
${ }_{5}^{5}$ Anne Marie BARON, Romans français du XIXe siècle à l'écran. Problèmes de l'adaptation, Presses Universitaires Blaise Pascal, Clermont Ferrand, 2008, p. 109-114.
} 
Michel Serceau, dans L'Adaptation cinématographique des textes littéraires: Théories et lectures, a particulièrement bien synthétisé les différentes théories qui se sont succédées ${ }^{6}$. Toutes ont voulu établir une " typologie de l'adaptation », le cinéma n'étant longtemps considéré que comme un simple «médium » dépendant de la Littérature. On a ainsi pu discerner deux catégories d'adaptations chez Etienne Fuzelier ${ }^{7}$ (l'adaptation passive et la transposition), puis trois avec André Bazin (l'adaptation, l'adaptation libre et " l'être esthétique nouveau »), auxquelles Claude Gauteur ${ }^{8}$ ajoute une quatrième, «l'œuvre d'auteur »'. En définitive, si la terminologie varie, on retrouve les mêmes constatations, seulement les points de vue diffèrent selon l'importance que l'on accorde à la «valeur » de l'adaptation. Ainsi André Garcia propose un tableau synthétique autour de deux notions et leurs variable « Faire la même chose (en moins bien, en aussi bien, en mieux) » ou bien « Faire autre chose (de moins bien, d'aussi bien, de mieux) $»^{10}$. Mais qu'entendon exactement par une bonne ou une mauvaise adaptation?

Michel Serceau cite Michel Mourlet qui montre bien que notre jugement est nécessairement faussé si l'on considère le cinéma uniquement dans un rapport de dépendance par rapport à la Littérature, dont il ne pourrait faire autre chose que la « vulgariser» : «Il n'y a pas de bonnes ou de mauvaises adaptations, il n'y a que du bon ou du mauvais cinéma ». Quel étalon faut-il adopter dans ce cas pour juger cette valeur?

Jeanne Marie Clerc, dans Littérature et Cinéma, entend quant à elle prendre en compte le point de vue quantitatif, sans jugement de valeur esthétique et réintroduire la notion essentielle de "spécificité » propre au visuel $^{11}$. La fidélité à l'œuvre originelle n'est plus le critère primordial, on s'intéresse à la qualité intrinsèque de la nouvelle création et à ce qu'elle ajoute à l'œuvre adaptée, en quoi elle la renouvelle. De plus de nombreux autres

\footnotetext{
${ }^{6}$ Michel SERCEAU, L'Adaptation cinématographique des textes littéraires : Théories et lectures, Editions du Céfal, Liège, 1999, 206 p.

${ }^{7}$ Etienne FUZELLIER, Cinéma et Littérature, Cerf, Paris, 1964, 325 p.

${ }^{8}$ Claude GAUTEUR, «Eloge de la spécificité », Cinéma et roman, Eléments d'appréciation, Revue des Lettres modernes, $\mathrm{n}^{\circ}$ 3638, 1958.

${ }^{9}$ Alain Garcia a lui aussi clairement synthétisé les différentes théories en proposant trois grands axes : l'adaptation, l'adaptation libre et la transposition : «L'adaptation est composée de l'illustration et de l'amplification ; l'adaptation libre de la digression et du commentaire ; quant à la transposition, elle est basée sur les principes de l'analogie et de l'écranisation ». L'adaptation du roman au film, IF Diffusion Dujaric, 1990, p. 21.

${ }^{10}$ Ibid., p. 262.

${ }^{11}$ Jeanne Marie CLERC, Littérature et cinéma, Nathan, 1993, 222 p.
} 
critères sont à prendre en considération, car comme le dit encore très justement Michel Serceau en conclusion :

L'adaptation [est] le produit d'une dialectique entre l'œuvre littéraire, le contexte socioéconomique de réalisation et les codes d'une culture. Toute adaptation témoigne d'une réception d'une œuvre littéraire. [...] On ne peut dans cette perspective s'en tenir aux relations immédiates et/ou explicites qu'elle entretient avec cette œuvre. Elle est inséparable du réseau d'œuvres littéraires et cinématographiques qui la précèdent, mais aussi celles qui sont produites dans le même champ historique et culturel. ${ }^{12}$

Dans le cadre de cette étude, nous n'avons pas eu d'autre prétention que de proposer un premier essai de synthèse qui mériterait d'être approfondi et complété par d'autres recherches complémentaires, et en particulier une étude de la réception, d'un point de vue synchronique et diachronique. De plus, nous nous sommes principalement reposée sur l'intrigue, pour avoir une base de comparaison, ce qui met nécessairement artificiellement l'accent sur le scénario et les dialogues, au détriment d'autres éléments tout aussi, voire plus signifiants, car spécifiquement liés au septième art (le cadrage, le montage, le travail de la lumière, le rôle de la musique, etc.).

Enfin, du fait que ces adaptations sont été aussi bien audiovisuelles que cinématographiques, pour avoir un jugement objectif, il faudrait plus précisément prendre en compte les spécificités de chacun de ces médias, qui répondent à des contraintes différentes susceptibles de considérablement modifier les volontés initiales du réalisateurscénariste. L'horizon d'attente du spectateur et du téléspectateur n'est pas forcément identique et la comparaison est parfois délicate, car le réalisateur de cinéma est souvent plus libre, en tant qu' « auteur », par rapport au réalisateur de télévision, en particulier depuis la récente dictature de «l'audimat », bien que les réalisateurs de cinéma aient été aussi limités par la censure des studios qui les produisaient, comme cela sera le cas, pour Dorothy Arzner.

Plus encore à la télévision qu'au cinéma, il est nécessaire de faire une distinction entre l'auteur unique et à part entière qu'est un écrivain, et un réalisateur, car un film est toujours le résultat du travail d'une équipe. Enfin, ce corpus inciterait également à explorer une autre dimension spatiotemporelle, car cette abondance d'adaptations dont la qualité est très variable, est toutefois une base d'étude passionnante pour mesurer les influences culturelles et surtout

\footnotetext{
${ }^{12}$ Michel SERCEAU, op. cit., p. 174.
} 
l'évolution historique de la représentation de la féminité dans l'imaginaire collectif.

Pour pouvoir pleinement comprendre les tenants et les aboutissants de la représentation de la féminité dans les diverses adaptations du roman de Zola, il convient tout d'abord de mesurer les ressemblances et les différences notables par rapport au roman, liées ou non aux spécificités du médium. Cellesci peuvent concerner l'intrigue, les caractères, l'atmosphère, la description, les dialogues, le message implicite ou explicite exprimé par Zola.

Si de nombreux chapitres et scènes du roman sont nécessairement supprimés pour les besoins de l'adaptation, qui peut difficilement avoir une longueur aussi importante que celle du roman, à part peut-être le long téléfilm de Cazeneuve (d'une durée de près de 6 heures), il est plus intéressant de repérer les passages qui ont été conservés, en particulier lorsque ceux-ci n'ont pas de rôle déterminant dans le déroulement de l'intrigue.

Outre les tableaux incontournables du roman comme la représentation de la «Blonde Vénus » au Théâtre des Variétés ou la course du Grand Prix de Paris au Bois de Boulogne, on retrouvera par exemple, dans toutes les adaptations la scène au cours de laquelle Nana humilie le comte Muffat en lui demandant tour à tour de faire le cheval ou le toutou : ce passage très « visuel» semble en effet synthétiser l'idée récurrente du roman, selon laquelle les hommes sont tels une meute de chiens autour de Nana, et parallèlement, cette scène pathéticoburlesque est censée symboliser le pouvoir total que Nana est parvenu à conquérir sur la gente masculine. Pouvoir discutable, puisqu'en l'occurrence, comme le sousentendent certaines adaptations, cette domination acceptée est finalement l'assouvissement d'un désir purement masculin.

Les scènes qui dévoilent le lesbianisme dans le roman, en revanche, ne sont que rarement reprises, ou bien de manière suggérée, comme dans le film de Molinaro, où Satin est nue au lit avec Nana, mais comme une bonne copine. Dans le téléfilm de Cazeneuve, la relation lesbienne est clairement évoquée, mais présentée de manière très pudique (on aperçoit les jambes de Satin et Nana qui s'entrecroisent dans le lit). Seul le film érotique de Dan Wolman représente franchement le lesbianisme et l'exploite. Cela s'explique sans aucun doute par le fait que le lesbianisme choque autant à l'époque de Zola que dans ses adaptations ultérieures, et même encore à notre époque, où la question apparaît encore taboue aux yeux de certains.

Ce qui est plus intéressant encore, c'est de remarquer que certaines scènes annexes ont malgré tout été conservées, selon la personnalité de chaque réalisateur, et en fonction du nouveau message qu'ils souhaitaient apporter. Ainsi, par exemple, Christian Jaque a choisi de nous montrer, comme dans le roman, Nana qui tient absolument à boire du lait dans une ferme, après sa nuit 
de fête, comme si elle cherchait à se purifier. Ou bien encore Renoir qui a voulu par exemple insister sur la débâcle des domestiques dans la maison de Nana, pour montrer le début de sa déchéance.

Pour pleinement comprendre tout l'intérêt de certains petits détails, pourtant très significatifs, qui ont été repris, il faudrait explorer plus en profondeur l'univers personnel très différents de ces divers réalisateurs, dont l'un des seuls points communs est finalement le goût pour les adaptations ${ }^{13}$. Bien souvent, l'œuvre adaptée n'est un tremplin pour l'imagination, comme l'écrivait André Bazin, les différences de structures esthétiques entre le roman et le film « requièrent d'autant plus d'invention et d'imagination de la part du cinéaste $»^{14}$, lors de la recherche des équivalences.

Pour ne donner que quelques exemples, Nana sera l'occasion pour Renoir, comme pour Christian Jaque, d'offrir un beau rôle à leur épouse respective, Catherine Hessling et Martine Carol, qui permettra de les sublimer, principalement par la mise en valeur de leur beauté physique. Renoir se ruinera en dépensant un million de francs pour ce premier film autoproduit qui ne rencontra pas le succès financier escompté ${ }^{15}$. Martine Carol, quant à elle, surtout connue pour le succès de Caroline Chérie, imprègnera largement le personnage de Nana par ce rôle préexistant. De même, il est aisé de reconnaître l'empreinte de Cazeneuve, réalisateur de télévision qui a contribué à faire de l'adaptation un "genre ", à travers des réalisations toujours très fidèles à l'intrigue romanesque, mais se rapprochant d'un « théâtre filmé », à l'opposé extrême de Molinaro, qui n'hésite pas à oblitérer aussi bien le tragique (ainsi Gervaise n'est pas morte, la blanchisseuse est devenue une femme de ménage

\footnotetext{
${ }^{13}$ Pour ne donner que quelques exemples représentatifs, Renoir a notamment adapté Madame Bovary (1933), Partie de campagne (1936), La Bête humaine (1938), Le Journal d'une femme de chambre (1946) ; Dorothy Arzner a réalisé en 1936 Craig's Wife, adapté de la pièce de théâtre de l'auteur populaire Vera Caspary ; Christian Jaque a adapté entre autres Carmen (1945), Boule de suif (1945), La Chartreuse de Parme (1948); Maurice Cazeneuve, Eugénie Grandet (1956), Illusions perdues (1966), Splendeurs et misères des courtisanes (1975); Dan Wolman avait adapté en 1975 My Michael d'après le roman d'Amos Oz et récemment Gei Oni (Valley of Strength) (2010), d'après le roman de Shulamit Lapid ; enfin, Edouard Molinaro a obtenu tardivement la reconnaissance, grâce à l'adaptation du Souper (1992) et la biographie de Beaumarchais, Beaumarchais, l'insolent (1996), mais aussi La Pitié dangereuse d'après Zweig (1979), «L'amour maudit de Leisenbohg» d'Arthur Schnitzler (1990) et Ce que voulait Maisie d'après Henry James (1995).

${ }^{14}$ André Bazin, «Pour un cinéma impur. Défense de l'adaptation », Qu'estce que le cinéma ?, Editions du CERF, 2010, Chapitre VIII, p. 95.

${ }^{15}$ Georges Sadoul note à ce sujet : « Il y eut un net succès public et critique, mais non financier, par la faute des distributeurs. », Dictionnaire des films, Nouvelle édition revue et augmentée par Emile BRETON, Microcosme / Seuil, 1990, p. 222.
} 
qui travaille de nuit dans les bureaux d'une entreprise), que le burlesque, pour proposer un drame psychologique personnel qui réinterprète l'histoire de Nana comme un parcours initiatique.

Au sujet des écarts par rapport à la trame originelle du roman, ce qui est bien entendu le plus manifeste, et surtout le plus significatif concernant le message que les cinéastes souhaitent donner à leur adaptation, c'est la fin de Nana.

Seules les adaptations de Renoir, et plus encore de Cazeneuve, qui suit l'intrigue du roman avec une méticuleuse fidélité, conservent la fin " moralisante » voulue par Zola ${ }^{16}$ : Nana agonise défigurée par la petite vérole dans une chambre d'hôtel. Fin, qui aurait été inspirée à Zola par la mort de la Cousine Bette de Balzac, selon Anne-Marie Baron, mais qui se rapproche également beaucoup, selon nous, de la fin de la grande libertine, Mme de Merteuil, dans Les Liaisons dangereuses. Or, dans le film de Renoir, la maladie semble être une métaphore des remords qui assaillent Nana, qui ne peut échapper à la culpabilité, et qui voit en hallucination tous ses amants morts. Renoir a choisi d'accentuer le contraste entre le comique qui nait de scènes burlesques et la fin dramatique, notamment par le passage du sépia au noir et blanc. Seul Cazeneuve a osé présenter crûment la décrépitude physique de Nana défigurée, sans aucun voile. Dans un cas le châtiment est moral et intérieur, à l'instar des tragédies grecques, Nana semble poursuivie par les Érinyes, déesses de la vengeance qui lui rappellent ses crimes jusqu'à lui faire perdre la raison, tandis que dans le second, le châtiment est physique et réaliste : les femmes de petite vertu avaient de grande chance de mourir d'une maladie transmise par l'un de leurs clients... La Nana de Renoir est libre comme la Carmen de Mérimée, elle n'a aucune excuse, à part la jouissance. Elle se moque en effet de tout et de tous, boit et fume comme un homme, et lance fièrement au comte Muffat que «l'argent, je crache dessus », comme elle le montre, dans une scène où elle s'amuse à jouer au billard avec des bibelots en porcelaine offerts par Georges.

En revanche, toutes les autres adaptations refusent cette fin qui a pu être jugée plutôt sordide et fortement moralisatrice. La plupart des réalisateurs ont toutefois choisi de conserver une fin «tragique », mais avec des nuances non négligeables.

Ainsi, dans le film d'Arzner, Nana choisit de se suicider, afin d'éviter aux deux frères qui l'aiment de s'entredéchirer, tandis que dans le film de

\footnotetext{
${ }^{16}$ Zola évoquait dans Le Voltaire du 28 octobre 1880 : « tout cet enguirlandage du vice, que je trouve dangereux pour les mœurs et d'une influence désastreuse sur les imaginations de nos filles pauvres. Je mets là la morale ; d'autres la mettent ailleurs. »
} 
Christian Jaque, c'est Muffat, incarné par Charles Boyer, qui commet un crime passionnel en étranglant Nana après l'avoir appelé " Ordure, mon amour », dans une dernière scène très théâtrale, cette fois peutêtre plus proche des tragédies shakespearienne, comme l'avait déjà suggéré Anna Gural Migdal.

Enfin, deux adaptations s'écartent encore plus radicalement de la fin du roman pour proposer une « happy end », c'est le cas du film de Molinaro, où Nana finit par récupérer son fils Loulou (qui était sa seule obsession au cours de l'histoire). La dernière image du film nous la montre enlacée dans les bras de Philippe, le fils du comte Muffat, qui de son côté accepte et encourage cette nouvelle union, après être retourné auprès de sa femme, ayant traversé une simple et très banale (pour notre époque) « crise de couple ». Tandis que dans le film de Wolman, Nana décide de partir méditer aux Indes et s'enfuit vers d'autres horizons à bord d'une montgolfière, un amant caché sous ses jupons, tandis que tous les protagonistes restés au sol l'applaudissent...

Si la suppression de certains personnages secondaires et la fusion d'autres, par principe d'économie, est quasiment un passage obligé pour les adaptations des romans populaires de grande envergure, ce qui nous intéresse surtout ici, c'est d'observer comment les auteurs et scénaristes gèrent et exploitent cette contrainte, car les choix de condensation ne sont jamais similaires. En effet, en dehors de quelques personnages féminins secondaires qui ont pu être aisément supprimés, souvent parce qu'ils faisaient double emploi (c'est notamment le cas des nombreuses actricescourtisanes que fréquente Nana), ce sont surtout les personnages masculins, les innombrables amants de Nana qui ont été fusionnés : deux personnages en un (comme Daguenet et Georges Hugon, qui représentent les « jeunes amants » inexpérimentés), ou bien dont les caractéristiques ont été transposées sur d'autres rôles.

Il est remarquable que la quasi-totalité des adaptateurs ont tenu à représenter une rivalité presque incestueuse entre deux hommes qui veulent avoir l'exclusivité des faveurs de Nana. Dans le roman de Zola, il s'agissait des deux frères Hugon : le cadet, Georges Hugon que Nana appelle «bébé », alors qu'il est quasiment du même âge qu'elle, et l'aîné, le lieutenant Philippe, qui tombe sous son charme, alors qu'il venait arracher son jeune frère des griffes de cette dernière. Cette rivalité fraternelle a été reprise dans plusieurs films, mais les personnages ont parfois été changés. Ainsi, dans le film d'Arzner, dans lequel la plupart des personnages masculins sont des militaires, il s'agit du Colonel André Muffat et de son frère le Lieutenant George Muffat (seul le prénom a été retenu). Dans le film de Renoir, il s'agit de Vandoeuvres et son neveu Georges Hugon (ici le nom complet a été conservé). D'autres adaptations ont reporté cette rivalité incestueuse sur une dualité père/fils : ainsi, dans le film de Wolman, il s'agit de Muffat et de son fils Hector (sa fille Estelle 
ayant disparue), et chez Molinaro, Nana s'éprendra du fils de Muffat, Philippe Muffat qui récupère l'uniforme de militaire du Philippe Hugon du roman.

On pourrait en conclure que finalement les principaux traits intemporels et universels conservés sont la rivalité amoureuse au sein d'une même famille, la différence d'âge (entre l'homme-père et l'homme-amant), et le personnage du militaire, qui permet quelle que soit l'époque de faire un rapprochement implicite ou explicite avec l'époque contemporaine du réalisateur : que l'on évoque la guerre franco-prussienne, la seconde guerre mondiale, ou bien le plus récent conflit dans les Balkans. C'est toutefois dans le film de Dorothy Arzner que les militaires sont les plus omniprésents, comme s'ils représentaient un entre-deux, un entre-deux-guerres, entre la puissance représentée par les aristocrates décadents du XIXe siècle et le pouvoir politicoéconomique des industriels et des commerçants qui tombent également à leur tour en décadence, dans le dernier film de Molinaro, au tout début du XXIe siècle...

Prendre le modèle de Nana pour étudier la représentation de la féminité pourrait sembler à la fois une évidence et un point de vue discutable, car réducteur. En effet, le personnage de Nana semble être la figure caricaturale d'une «féminité exacerbée », poussée à son extrême au point qu'elle ne semble plus réaliste. Les critiques, à la sortie du roman, n'ont d'ailleurs pas manqué de reprocher à Zola ce manque de réalisme, qui aurait été lié à un défaut de documentation. Cependant, il y a sans doute un parti pris volontaire qui a été perçu par les divers adaptateurs : Nana « surjoue » pour provoquer dans le roman comme dans beaucoup d'adaptations. On pourrait dire comme Anna Gural Migdal que la Nana de Zola « apparaît à la fois unique et universelle, l'incarnation synecdotique de son sexe poussée à ses limites, aux confins de l'indicible $»^{17}$. A l'écran, cela se traduit par le fait qu'elle est physiquement présente quasiment sur tous les plans et souvent en très gros plans, au point de finir, en particulier dans la première adaptation muette de Renoir, par accaparer l'écran jusqu'à l'obsession, voire l'écœurement, ce qui semble reproduire l'impression, sans doute voulue par Zola, que le lecteur peut ressentir à la lecture du roman.

Les autres constantes récurrentes que l'on retrouve dans les adaptations du roman sont les « attraits féminins » de Nana amplement soulignés, qui sont censés rendre fous les hommes qui l'entourent, et en particulier sa chevelure chatoyante (blonde, rousse ou brune), toujours présentée comme une crinière éblouissante. Les autres éléments physiques qui exacerbent sa féminité sont

\footnotetext{
${ }^{17}$ Anna GURAL MIGDAL, « Nana, figure de l'entre et de l'autre », dans L'Ecriture du féminin chez Zola et dans la fiction naturaliste / Writing the Feminine in Zola and Naturalist Fiction, Peter Lang, Bern, 2003, p. 313.
} 
son maquillage travaillé, ses seins qu'elle dévoile aisément, mais aussi et surtout son postérieur, comparé dans le roman à la croupe d'une jument, et très souvent mis en avant à l'écran par les costumes portées par les actrices, en particulier Véronique Genest, la Nana pétillante de Maurice Cazeneuve, qui use abondamment du fameux déhanché, à la fois simple et étudié, qui permet à lui seul de terrasser la volonté des hommes...

Dans le roman de Zola, Nana n'était pas «belle » à proprement parler, aux yeux des autres femmes, elle est même qualifiée de « grasse », mais aux yeux des hommes, sa sensualité voluptueuse est justement exprimée par son embonpoint, ses formes généreuses, qui comme sa peau clair, reflètent la vie, la fraîcheur, la bonne santé et le plaisir de la chair. Edouard Molinaro fait encore une fois exception en choisissant comme héroïne Lou Douillon, une actrice filiforme, qui joue plutôt sur un charme androgyne.

Les autres traits, psychologiques cette fois, qui semblent caractériser la « femme » en général, représentée par Nana, et non seulement la « prostituée », sont sa frivolité, sa bêtise, son ignorance et sa naïveté. Les amants de Nana, lui pardonnent aisément ces défauts, qu'ils considèrent au contraire encore une fois comme des qualités qui accentuent son charme d' " adorable ingénue », qui sait être en même temps une « diablesse de volupté ». Elle est successivement l'incarnation de tous les fantasmes masculins : la femme-enfant, la femmeanimale, l'inhumaine. En revanche, dans le roman du moins, elle ne peut prétendre au stéréotype de la femme-mère (bien qu'ayant un fils, elle ne fait que singer les comportements d'une mère), ni celle de la femme-victime (car elle est pleinement responsable des malheurs qui lui arrivent).

Chez Zola, contrairement à ce que montrent certaines adaptations, on ne trouve aucune forme de pitié ou de compassion concernant la condition féminine, et en particulier la condition des prostituées, mais le roman a suscité une réaction des lecteurs-adaptateurs, qui ont relu le roman à la lumière de leur propre époque, et qui ont voulu exploiter les éléments intemporels du roman de Zola, tout en apportant un nouveau message et une nouvelle morale.

Le film d'Arzner semble être le premier film qui montre une forme de révolte face à la vision naturaliste de Zola. C'est le début d'une revendication féministe que l'on découvre dans le film d'une réalisatrice pionnière qui fut la première femme à faire carrière dans le système des studios hollywoodiens. Le film commence par l'enterrement de Gervaise comme une indigente. On assiste alors à une scène au cours de laquelle Nana déclame ses premières revendications féministes à un couple de paysans, tout en lessivant le sol de leur pauvre masure, scène qui fait nécessairement penser à une illustration du séjour de Cosette chez les Thénardier, dans Les Misérables d'Hugo... Lorsque 
la vieille lui prédit qu'elle finira comme sa mère, comme une « mauvaise femme », elle réplique :

Ma mère n'était pas mauvaise, elle était faible. Oui, faible ! Oh, ce sont les hommes qui font des femmes ce qu'elles sont. Je ne sais pas encore ce que je vais devenir, mais je ne serai pas faible et je ne serai pas pauvre!

Et effectivement, quelques années plus tard, on la retrouve dans un café empli de militaires, en train de bousculer dans une fontaine un soldat qui lui avait manqué de respect, en la courtisant sans façons. Mais sa revendication féministe ne va pas beaucoup plus loin. On sait que le film a subi la censure, il faudrait donc approfondir l'étude des conditions de réalisation, pour pouvoir pleinement juger ce qui est ou non lié à la volonté de la réalisatrice Dorothy Arzner, qui aurait travaillé en collaboration avec George Fitzmaurice. On sait en effet que le producteur Samuel Goldwyn voulait, par cette adaptation, lancer la carrière de la starlette Anna Sten, une «nouvelle Garbo », mais le film n'eut pas le succès attendu. Dans la suite du film, Nana perd petit à petit sa révolte. Elle est façonnée par Gaston Greiner, le producteur (qui remplace Bordenave) et devient un personnage un peu anachronique, à l'image d'une Marlène Dietrich, dans L'Ange bleu, que l'on voit exécuter un show sensuel, en chantant pour des soldats, fumant et se déhanchant lascivement. Elle est finalement atteinte du mal qui semble être la principale faille des femmes : «l'amour ». Elle tombe en effet éperdument amoureuse du lieutenant Georges Muffat, qui est mobilisé et contraint de s'éloigner d'elle. Son frère aîné, le Colonel André Muffat s'éprend de Nana, alors qu'il venait lui proposer une transaction financière pour qu'elle se sépare de son frère. Nana cède, mais lorsque les deux frères apprennent la double relation qu'elle a entretenue, elle décide brutalement de se suicider pour éviter que les frères ne s'entredéchirent... Nana apparaît comme une victime de l'amour et s'éloigne définitivement de la figure d'une courtisane vénale.

On pourrait également parler d'une forme de revendication féministe, dans le film de Dan Wolman, qui pourrait apparaître comme un OVNI dans ce corpus. En effet, cette production classée par Anne Marie Baron dans la catégorie des « pornos soft », n'est que rarement mentionnée lorsqu' on évoque les adaptations de Nana. Elle l'évoque cependant très brièvement en la jugeant « assez fidèle au canevas de l'intrigue », en «y intercal[ant] de nombreuses 
scènes purement érotiques. ${ }^{18}$. Ce film ne fait que jouer à fond, selon elle, sur la composante érotique du roman en termes de marketing cinématographique. Cependant, Anna Gural Migdal, qui a par ailleurs étudié la représentation du féminin dans les romans de Zola, a trouvé cette adaptation suffisamment intéressante pour y consacrer un article intitulé « Nana (1982) de Dan Wolman ou la naissance du cinéma ${ }^{19}$. Il est vrai que la distribution, qui fait apparaître au côté de Nadia Berger, l'acteur français de renom, JeanPierre Aumont, présage à elle seule que cette production n'est pas qu'un simple film de série B. L'originalité de cette adaptation érotique du roman, réalisée par un cinéaste israélien, auteur de films indépendants peu connus en France, tient au fait que Nana n'est plus une actrice de théâtre, mais une stripteaseuse, qui participe au début du cinéma aux côtés de Méliès, devenu réalisateur de films pornographiques. Cette composante érotique est bien présente dans le roman de Zola, ce qui explique que le roman avait d'ailleurs donné lieu en 1970 à une première adaptation érotique par Mac Ahlberg ${ }^{20}$ moins remarquée. L'un des leitmotivs intéressants du film de Wolman est le topic du voyeurisme mis en abyme : les spectateurs deviennent des voyeurs observant des personnages qui sont euxmêmes aussi des voyeurs. A plusieurs reprises, un trou aménagé dans le mur derrière un tableau pour épier la loge de Nana, permettra à des vieillards de l'observer se baigner, ou bien à Hector de la regarder avoir des rapports sexuels avec ses concurrents, Gérard, ou Steiner. Nana devient également une voyeuse, lors d'une chasse à cour sexuelle qu'elle observe à la lorgnette. Cela donne lieu à des plans de nus souvent plus picturaux que réellement «pornographiques ».

Une autre composante du roman de Zola qui est particulièrement bien représenté dans le film est le côté burlesque de Nana qui avait également été pleinement exploité dans le film de Renoir, en revanche, tous les aspects dramatiques et tragiques ont été effacés. Nana devient un chantre de la liberté sexuelle. Elle va jusqu' au bout de ses envies, sans que ses actions ne portent réellement à conséquence. Le film est emprunt de la vague de libération sexuelle des années 70. Ainsi tout est traité sur le mode burlesque et léger, comme lorsque Nana vient enlever Hector au moment de son mariage, en lui montrant ses seins, et qu'ils finissent par faire l'amour dans une calèche, alors

\footnotetext{
${ }^{18}$ Anne Marie BARON, « Nana, cet objet obscur du plaisir », op. cit., p. 109.

${ }^{19}$ Anna GURAL MIGDAL, L'écrité cran des «Rougon Macquart » : conceptions iconiques et filmiques du roman chez Zola, Presses universitaires du Septentrion, Villeneuve d'Ascq, 2012, chapitre IX, p. 221-251.

${ }^{20}$ Ce film francosuédois intitulé, Poupée d'amour, présenterait un «portrait au vitriol de la haute bourgeoisie ».
} 
que tous les invités de la noce tentent de les rattraper comme dans de nombreux films comiques du cinéma muet, ou encore lorsque, comme nous l'avons dit, Nana décide de s'enfuir en montgolfière à la fin du film.

Enfin, le film le plus récent de Molinaro est peutêtre l'adaptation (quel que soit le jugement de valeur que l'on porte sur la qualité de celleci) qui invite le plus les spectateurs à réfléchir sur le jeu des transpositions. En effet, il y a clairement deux aspects notables dans cette dernière : d'une part la volonté de reprendre une composante essentielle du roman de Zola qui est la description d'une bourgeoisie en déclin, en dénonçant le pouvoir de la religion ${ }^{21}$, de l'argent et de la politique, tout en la transposant à notre époque, et d'autre part le désir d'offrir au personnage de Nana un destin radicalement différent de celui que Zola lui avait dévolu.

Le monde des affaires, la compromission des politiciens et le rôle du sexe et du pouvoir dans la vie politicoéconomique de notre époque ont été particulièrement bien représentés dans le film de Molinaro. En revanche, pour proposer un autre parcours possible de Nana, le réalisateur a dû considérablement s'écarter du roman et du message voulu par Zola. Dans le film, Nana est dès le début présentée comme une victime : celle d'un viol collectif à la suite duquel elle a accouché d'un fils qui a dû être placé à la DASS. Son combat, tout au long du film va être motivé par l'unique obsession de parvenir à récupérer la garde de son fils. Ce ne sont pas les hommes uniquement (certains l'aideront même finalement plus que les femmes, à l'inverse notamment de l'assistante sociale que Nana finira par passer à tabac), mais toute une société qui est responsable des extrémités auxquelles elle est contrainte et qu'elle tente vainement d'éviter. Mais cette volonté de totalement déculpabiliser le personnage de Nana, produit des scènes peu vraisemblables. Ainsi, Nana n'est pas une véritable prostituée, elle ne fait que masturber des hommes pour de l'argent, car elle ne peut coucher, affirmetelle naïvement (au vu de son comportement général), qu'avec les hommes qu'elle aime... Ainsi le personnage de Vandoeuvres, interprété par Jean Claude Brialy, n'aura jamais de relations sexuelles avec elle, en revanche il exploitera l'attraction sexuelle qu'elle exerce sur les hommes, pour parvenir à ses fins, en faisant chanter Muffat, grâce à des photos compromettantes ; mais en définitive, seul le pouvoir l'intéresse et non le sexe. Très curieusement dans cette adaptation qui devrait ne plus dépendre de la censure d'antan (la nudité et

\footnotetext{
${ }^{21}$ Zola avait ainsi décrit son « sujet philosophique » dans l'ébauche du roman « toute une société se ruant sur le cul. Une meute derrière une chienne, qui n'est pas en chaleur et qui se moque des chiens qui la suivent. Le poème des désirs du mâle, le grand levier qui remue le monde. Il n'y a que le cul et la religion ». Ebauche du roman, BNF, NAF, Ms. 10313, fos 207-208.
} 
la sexualité étant désormais couramment présentés sans voile), est pourtant le film qui masque le plus la sexualité débridée et assumée de Nana.

Nana devient une courtisane presque malgré elle, toujours à contrecœur. Et si elle couche avec quelques jeunes hommes, ce ne sont pour elle que des « copains avec qui on couche », des « sex friends », comme on pourrait dire aujourd'hui. Or, comme dans le film d'Arzner, où le seul tort de Nana est d'être touchée par l'amour : Nadia Coupeau tombera elle aussi éperdument amoureuse de Philippe, le fils de Muffat. C'est à ce moment-là que l'histoire pourrait tourner en tragédie : Muffat a un accident de voiture, après avoir appris que son fils est l'amant de Nana. Pourtant, comme on l'a dit, le film se termine en happy end: Muffat pardonne et retourne avec son épouse Sabine, Nana récupère son fils, devient une « honnête femme » que l'on voit en train de diriger une chorale d'enfants à Noël. La dernière image du film nous la montre enlacée dans les bras de Philippe venu la rejoindre, ayant lui aussi tout pardonné.

La victoire de Nana a cependant été payée bien cher, et c'est plus la victoire d'une « mère courage » que celle d'une femme qui a su prouver son indépendance... Schématiquement, on pourrait dire que si dans le film de Renoir, comme dans celui de Cazeneuve, Nana est une femme « forte » par son pouvoir de manipulation sur les hommes, tandis qu'elle est une victime de l'amour dans le film de ChristianJaque et celui de Dorothy Arzner, ou une victime de la société dans celui de Molinaro, elle n'apparaît finalement comme une femme que l'on pourrait qualifier de « libre et indépendante » que dans la version de Wolman. Cependant cette liberté est tout de même là aussi limitée, puisqu'elle ne s'affirme finalement que dans les limites de la réalisation de fantasmes qui restent masculins...

Les quelques éléments de réflexion que nous venons de proposer ne peuvent donner qu'une vision incomplète de la portée des diverses adaptations de ce roman. Comme nous l'avons dit, il aurait en effet fallu, ce que la taille de cette étude ne permettait pas, étudier la réception de ces productions par rapport à celle du roman. Notre objectif n'était toutefois que d'envisager un aspect très particulier, celui du traitement de la féminité à travers des créations plus ou moins éloignées de la source d'inspiration. En tant que chercheur en Littérature qu'estce qui nous importe le plus finalement?

Les critiques ont abandonné depuis longtemps le simple «étalon » de la fidélité à l'œuvre adaptée, tous s'accordent sur le fait qu'une adaptation ne doit pas clôturer, mais au contraire ouvrir le roman support, en proposant une réflexion présente dans l'œuvre originelle, mais que les critiques littéraires non pas toujours perçue, et que l'œil nouveau d'un cinéaste peut exploiter. Le principal, pour que la création cinématographique ou télévisuelle soit réussie 
aux yeux du grand public, comme aux yeux des chercheurs en quête de matière à réflexion, c'est que l'adaptation acquière une existence propre, en dehors d'une simple illustration, ce qui ne constitue pas nécessairement l'assurance que le spectateur ait envie de plonger ou de revenir au roman d'origine. L'originalité de ce roman, c'est qu'il semble avoir la capacité de facilement se prêter à la transformation générique : Nana n'est plus qu'un roman, adapté très tôt en pièce de théâtre par l'auteur, c'est un tableau (la « Nana rêvée » de Manet présentée par le narrateur Fauchery dans le téléfilm de Cazeneuve), un film, un téléfilm, un feuilleton, une parodie ${ }^{22}:$ l'œuvre s'est enrichie de tous ces prolongements qui alimentent un mythe originel constamment renouvelé en fonction des époques et des pays : celui d'une femme réelle, débordante de vie. Nana n'est ni bonne ni mauvaise, ce qui lui permet, au-delà des idéalisations, d'échapper aux clichés traditionnels de la femme nécessairement ange ou démon. Nana n'est plus la simple « cocotte » qui utilise à mauvais escient ses aouts féminins pour tenter vainement de progresser les échelons de la société, elle est devenue une femme amoureuse qui assume et use de sa féminité pour affirmer non pas sa supériorité, mais son égalité. Cependant pour revendiquer cette égalité, elle aura dû perdre certains des stéréotypes qui semblaient constitutifs de sa féminité. C'est en ce sens que nous interpréterons l'androgénisation ${ }^{23}$ de la Nana de Molinaro qui finira par dire à Satin : « J'ai peur, mais je te jure que je n'aurai jamais plus peur. Il ne faut pas, il faut qu'on soit comme des mecs, il faut avoir une paire de couilles comme eux »...

\section{Annexe 1 : Distribution des adaptations de Nana étudiées}

Nana, film français muet en noir et blanc, sorti au cinéma en 1926, réalisé et produit par Jean Renoir, scénario de Pierre Lestringuez et Jean Renoir, avec Catherine Hessling (Nana), Jean Angelo (Le comte de Vandœuvres), Werner Krauss (Le comte Muffat), Raymond GuérinCatelain (Georges Hugon), Claude Moore (Claude Autant Lara) (Fauchery), Pierre Champagne (Hector de la Faloise), Jacqueline Ford (Rose Mignon), Jacqueline Forzane (La comtesse

\footnotetext{
${ }^{22}$ Nous faisons allusion à l'opéretteparodie, « Nana et compagnie », étudiée par Daniel Compère, dans Naturalisme et excès visuels: pantomime, parodie, image, fête. Mélanges en l'honneur de David Baguley, Cambridge Scholars Publishing, Newcastle upon Tyne, 2009, p. 61-68.

${ }^{23}$ Cet aspect androgyne était d'ailleurs bien présent dans le roman, comme le souligne encore Anna Gural Migdal, qui emploie la notion d'un « entregenre » qui s'exprime notamment par le travestissement, l'androgynie ou la bisexualité de Nana : «les stéréotypes de la féminité se conjuguent à ceux de la masculinité », « Nana, figure de l'entre et de l'autre », op. cit., p. 316.
} 
Sabine Muffat), Valeska Gert (Zoé), René Koval (Fontan), Pierre Philippe (Pierre Lestringuez) (Bordenave). Durée : 2H30.

Nana, film américain en noir et blanc, sorti au cinéma en 1934, produit par Samuel Goldwyn, réalisé par Dorothy Arzner et George Fitzmaurice [ce dernier n'apparaît pas au générique], scénario d'Harry Wagstaff Gribble et Willard Mack, image de Gregg Toland, musique d'Alfred Newman, avec Avec Anna Sten (Nana), Lionel Atwill ( le colonel André Muffat), Richard Bennett (Gaston Greiner), Mae Clarke (Satin), Phillips Holmes (le lieutenant George Muffat), Muriel Kirkland (Mimi), Reginald Owen (Bordenave), Helen Freeman (Sabine Muffat), Lawrence Grant (le grand duc Alexis), Jessie Ralph (Zoé). Durée 1H30.

Nana, production franco-italienne des Films Jacques Roitfeld (Paris) et Cigno Films (Rome), réalisé en 1954, sorti au cinéma en France en 1955, réalisé par ChristianJaque, scénario écrit par Jean Ferry, ChristianJaque, Albert Valentin, Henri Jeanson, dialogues d' Henri Jeanson, avec Martine Carol (Nana), Charles Boyer (Le comte Muffat), Jacques Castelot (Le duc de Vandeuvres), Jean Debucourt (Napoléon III), Walter Chiari (Fontan), Noël Roquevert (Le banquier Steiner), Dora Doll (Rose Mignon), Elisa Cegani (La comtesse Sabine Muffat), Paul Frankeur ( Bordenave), Dario Michaelis (Fauchery), Daniel Ceccaldi (Le lieutenant Philippe Hugon), Marguerite Pierry (Zoé), Luisella Boni (Estelle Muffat). Durée 2H00.

Nana, téléfilm français en 4 épisodes de 90 min. diffusé sur France 2 en 1981, réalisé par Maurice Cazeneuve, avec Véronique Genest (Nana), Guy Tréjan (Comte Muffat), Patrick Préjean (Fontan), Sacha Briquet (Comte de Vandeuvres), Tony Rödel (Steiner), Marion Game (Zoé), Micky Sébastian (Satin), Vincent Ropion (Georges Hugon), Albert Simono (Labordette), André Cellier (Bordenave), Armand Mestral (Mignon), Sarah Sanders (Sabine), Charlotte de Turckheim (Rose), Madeleine Barbulée (Mme Lerat). Durée : 5H50.

Nana, [titre français : Nana, Le désir], réalisé en 1982, sorti au cinéma en 1983, coproduction américanoitalienne de Menahem Golan et Yoram Globus, réalisé par Dan Wolman, scénario de Marc Behm, musique d'Ennio Morricone, avec Katya Berger (Nana), Jean Pierre Aumont (comte Muffat), Yehuda Efroni (Steiner), Mandy Rice Davies (Sabine), Massimo Serato (Faucherie), Debra Berger (Satin), Shirin Taylor (Zoé), Annie Belle (Rennée de Chéselles), Paul Muller (Xavier), Marcus Beresford (Hector Muffat), Robert Bridges (Fontan), Tom Felleghy (Georges Méliès). Durée : $1 \mathrm{H} 30$.

Nadia Coupeau, dite Nana, téléfilm français en 2 épisodes diffusé sur France 2 en 2001, réalisé par Edouard Molinaro, scénario d'Edouard Molinaro et 
Olga Vincent, avec Lou Doillon (Nana), Bernard Le Coq (Paul Muffat), Assumpta Serna (Sabine Muffat), Ticky Holgado (Jean Bordenave), Jean Claude Brialy (Vandoeuvres), Jocelyn Quivrin (Philippe Muffat), Vincent Martinez (Luc Faugier), Idwig Stephane (Steiner), Jauris Casanova (Daguenet), Linda Bouhenni (Zoé), Serge Kribus (Laborde), Tomer Sisley (Fontan). Durée : 3H25.

\section{Annexe 2: Liste des adaptations de $\mathrm{Nana}^{24}$}

1910. Nana (Storstadens Hyaene, «La Hyène de la Capitale »). Réalisation / Knud Lumbye. Danemark. Interprétation : Ellen Lumbye (Nana), Schioler Link (Muffat). [Daté de 1912 selon Anne Marie Baron...]

1914. Nana. Réalisation : Camillo de Riso, Ugo Pittei. Italie. Interprétation : Lila Pescatori (Nana). [Anne Marie Baron note que cette adaptation est anonyme...]

1916. Nana. Réalisation : Nino Martoglio. Interprétation : Tilda Kassay (Nana). [Anne Marie Baron note : 1917/1919. Nanà / Una donna funesta de Camillo de Riso, Italie. Interprétation : Tilde Kassay].

1917. A Man and a Woman. Réalisation : Alice Blaché [Anne Marie Baron note Herbert Blaché et Alice Guy ?]. Etats Unis. Interprétation : Edith Hallor (Nana).

1926. Nana. Réalisation : Jean Renoir. France. Scénario de Pierre Lestringuez.

Décors de Claude Autant Lara. Les soustitres ont été rédigés par Denise Le Blond Zola. Interprétation : Catherine Hessling (Nana), Werner Krauss (Muffat), Jean Angelo Vandeuvres), Raymond Guérin Catelain (Georges Hugon), Jacqueline Forzane (comtesse Muffat), Valeska Gert (Zoé), Harbacher (Francis, le coiffeur de Nana).

1934. Nana (Lady of the Boulevards). Réalisation : Dorothy Azner [et George

Fitzmaurice selon Anne Marie Baron]. Etats Unis. Interprétation : Anna Sten (Nana), Lionel Atwill, Richard Bennet, Philippe Hilmes, Mae Clarke. 1943. Nana. Réalisation : Celestino Gorostiza [et Roberto Galvadon selon Anne-Marie Baron]. Mexique. Interprétation : Lupe Vélez (Nana), Miguel Angel Ferriz, Chela de Castro.

${ }^{24}$ Liste réalisée à partir du Guide Emile Zola d'Alain PAGES et Owen MORGAN (Ellipses, 2002), complétée par l'ouvrage d'Anne Marie BARON, Romans français du XIX ${ }^{e}$ siècle à l'écran. Problème de l'adaptation, op. cit. Les ajouts de cette dernière sont indiqués entre crochets. 
1955. Nana. Réalisation : Christian Jaque. France. Dialogues de Henri Jeanson. Interprétation : Martine Carol (Nana), Charles Boyer (Muffat), Dora Doll, Jacques Castelot, Noël Roquevert, Jean Debucourt, Pierre Palau.

1968. Nana, téléfilm, Grande Bretagne, 1968. Interprétation : Katherine Schofield.

1971. Nana. [Anne Marie Baron ajoute : Nana 70 (Poupée d'amour / Tag mejälska mej...)] Réalisation : Marc Ahlberg. Suède [/France ?]. Interprétation : Anna Gael (Nana), Gillian Hills, Lars Lunoë.

1981. Nana. Téléfilm. Réalisation : Maurice Cazeneuve. France. Dramatique de télévision en quatre épisodes, diffusée sur Antenne 2. Interprétation : Véronique Genest (Nana), Guy Tréjean (Muffat).

1983. Nana. Réalisation : Dan Walman. Coproduction américanoitalienne. Interprétation : Katya Berger, JeanPierre Aumont, Mandy Rice Davies.

1985. Nana. Réalisation : Rafael Baledon [Zeledón selon Anne Marie Baron] et José Bolanos. Mexique. [Interprétation : Irma Serrano].

[1995. Nana, téléfilm de Miguel Alexandre, Danemark. Interprétation : Bernadette Heerwagen.]

[1997. Nana, de Claude Miller, France. Interprétation Emmanuelle Seigner.] [1999. Nanà, téléfilm d'Alberto Negrin, Italie. Interprétation : Francesca Dellera. ]

2001. Nadia Coupeau, dite Nana. Téléfilm en deux épisodes. Réalisation : Edouard Molinaro. Scénario d'Edouard Molinaro et Olga Vincent, d'après un récit de Catherine Rihoit « librement adapté du roman d'Emile Zola». Téléfilm en deux parties, diffusé sur France 2 les 26 et 27 novembre 2001. Interprétation : Lou Doillon (Nana), Bernard Le Coq (Paul Muffat), Linda Bouhenni (Zoé), Idwig Stéphane (Steiner), Ticky Holgado (Bordenave), Jean Claude Brialy (Vandoeuvres), Jauris Casanova (Daguenet), Tomer Sisley (Fontan). 